\title{
Characterization and functional analysis of the Hydroxycinnamoyl-CoA: shikimate hydroxycinnamoyl transferase (HCT) gene family in poplar
}

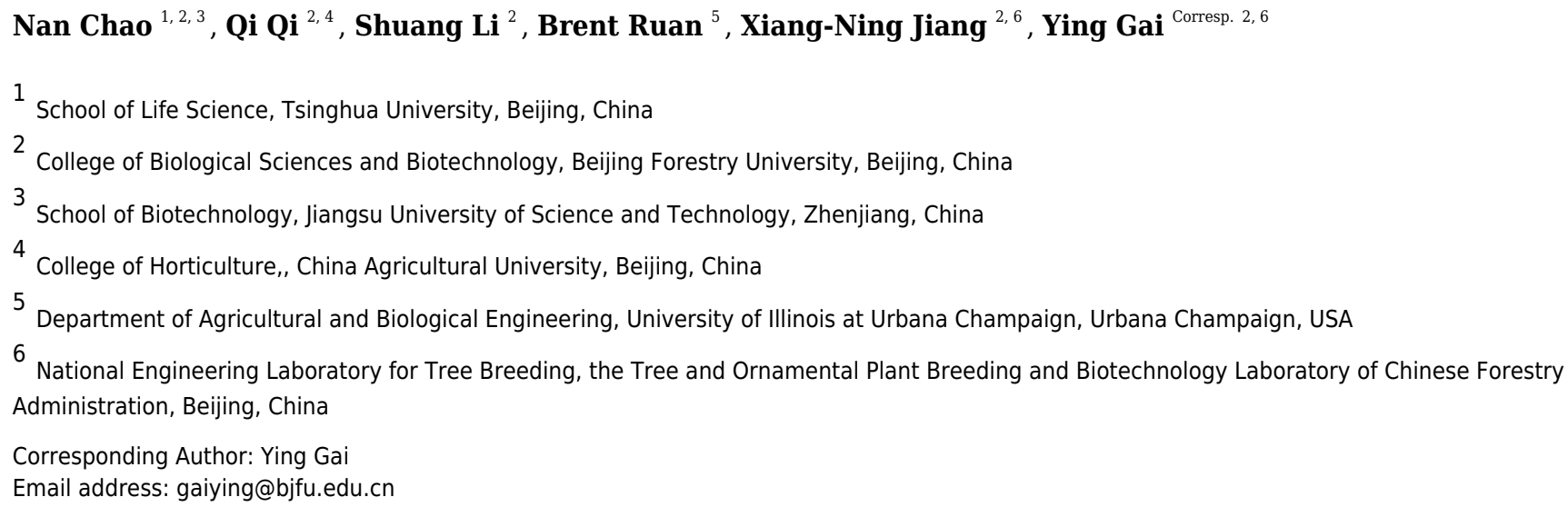

Hydroxycinnamoyl-CoA: shikimate hydroxycinnamoyl transferase (HCT) divides the mass flux to $\mathrm{H}, \mathrm{G}$ and $\mathrm{S}$ units in monolignol biosynthesis and affects lignin content. Ten HCT homologs were identified in the Populus trichocarpa (Torr. \& Gray) genome. Both genome duplication and tandem duplication resulted in the expansion of HCT orthologs in Populus. Comprehensive analysis including motif analysis, phylogenetic analysis, expression profiles and co-expression analysis revealed the divergence and putative function of these candidate PoptrHCTs. PoptrHCT1 and 2 were identified as likely involved in lignin biosynthesis. PoptrHCT9 and 10- are likely to be involved in plant development and the response to cold stress. Similar functional divergence was also identified in Populus tomentosa Carr. Enzymatic assay of PtoHCT1 showed that PtoHCT1 was able to synthesize caffeoyl shikimate using caffeoyl-CoA and shikimic acid as substrates. 
1

2

3 Nan Chao ${ }^{1,2,4}$, Qi $\mathbf{Q i}^{2,5}$, Li Shuang ${ }^{2}$, Brent Ruan ${ }^{6}$, Xiang-Ning Jiang ${ }^{2,3}$ and Ying Gai ${ }^{2,3 \dagger}$

$4 \quad{ }^{1}$ School of Life Science, Tsinghua University,

$5 \quad{ }^{2}$ College of Biological Sciences and Biotechnology, Beijing Forestry University,

$6{ }^{3}$ National Engineering Laboratory for Tree Breeding, the Tree and Ornamental Plant Breeding and

7 Biotechnology Laboratory of Chinese Forestry Administration, Beijing 100083, China,

$8 \quad{ }^{4}$ School of Biotechnology, Jiangsu University of Science and Technology,

$9{ }^{5}$ College of Horticulture, China Agricultural University,

${ }^{6}$ Department of Agricultural and Biological Engineering, University of Illinois at Urbana Champaign

11

12

13

14

$\dagger$ To whom correspondence should be addressed: Tel (Fax): +86 1062338063 ;

E-mail: gaiying@bjfu.edu.cn, Beijing Forestry University.

The English in this document has been checked by at least two professional editors, both native speakers of English, and the grammars were also checked using Grammarly. 
Abstract: Hydroxycinnamoyl-CoA: shikimate hydroxycinnamoyl transferase (HCT) divides the mass flux to H, $\mathrm{G}$ and $\mathrm{S}$ units in monolignol biosynthesis and affects lignin content. Ten HCT homologs were identified in the Populus trichocarpa (Torr. \& Gray) genome. Both genome duplication and tandem duplication resulted in the expansion of HCT orthologs in Populus. Comprehensive analysis including motif analysis, phylogenetic analysis, expression profiles and co-expression analysis revealed the divergence and putative function of these candidate PoptrHCTs. PoptrHCT1 and 2 were identified as likely involved in lignin biosynthesis. PoptrHCT9 and 10- are likely to be involved in plant development and the response to cold stress. Similar functional divergence was also identified in Populus tomentosa Carr. Enzymatic assay of PtoHCT1 showed that PtoHCT1 was able to synthesize caffeoyl shikimate using caffeoyl-CoA and shikimic acid as substrates.

Key words: Hydroxycinnamoyl- CoA: shikimate hydroxycinnamoyl transferase; enzymatic synthesis; divergence; gene family; monolignol; Populus

\section{Introduction}

Primary walls and secondary walls protect plant cells and define the shapes of cells, tissues, organs and ultimately the whole plant body (Zhong et al. 2019). Lignin is an important component for secondary cell walls and is one of the most abundant components of biomass in plants (Boerjan et al. 2003; Tang \& Tang 2014). Therefore, lignin plays a vital role in plant physiology. Owing to the recalcitrant chemical nature and the complexity of lignin, lignin limits the conversion efficiency of lignocellulosic biomass to ethanol (Poovaiah et al. 2014; Vanholme et al. 2010). Modifying trees to have less lignin or more-degradable lignin along with normal growth, can reduce the high processing costs and carbon footprint of making paper, biofuels, and chemicals (Ralph et al. 2019; Tang \& Tang 2014; Wang et al. 2019; Xu \& Li 2016; Zhao 2016).

The biosynthetic pathway for lignin has been studied extensively and the phenylpropane pathway which begins with phenylalanine, is responsible for monolignol biosynthesis. (Boerjan et al. 2003; Karkonen \& Koutaniemi 2010; Maeda 2016; Ralph et al. 2019; Vanholme et al. 2010; Wang et al. 2019; Xu \& Li 2016). Monolignol is the general name for lignin building blocks. Our understanding of the monolignol biosynthetic pathway has continued to grow, and now 11 enzyme families and 24 metabolites are associated with it (Vanholme et al. 2019). Hydroxycinnamoyl- CoA: shikimate hydroxycinnamoyl transferase (HCT) is located at a key point in the monolignol biosynthetic pathway and is conserved across all land plants. In conjunction with 
$43 \mathrm{C} 3 \mathrm{H}$ ( $p$-coumarate 3-hydroxylase), HCT catalyzes two steps to direct the mass flux from the $\mathrm{H}$ monolignol to $\mathrm{G}$

44

and S monolignols (Figure 1). HCT first catalyzes the coupling of $p$-coumaroyl-CoA with shikimate to produce p-coumaroyl shikimate (Hoffmann et al. 2004; Hoffmann et al. 2003). Caffeoyl shikimate is generated by C3H and is then transesterified by HCT to form caffeoyl-CoA. This reaction is probably reversible based on the reported in vitro activity (Lepelley et al. 2007; Wang et al. 2014). Caffeoyl shikimate esterase (CSE), a new member in monolignol biosynthesis pathway recently discovered in plants can hydrolyze caffeoyl shikimate to release caffeate (Ha et al. 2016; Saleme et al. 2017; Vanholme et al. 2013; Vargas et al. 2016). Although downregulation of HCT expression improves forage digestibility and saccharification efficiency, it negatively affects plant growth resulting in shorter plants (Li et al. 2010; Shadle et al. 2007).

HCT (GO:0102660) belongs to the BAHD acyltransferase family and is able to utilize many non-native substrates. Some HCTs (also called HQT) can use quinate as a substrate in addition to shikimate (Eudes et al. 2016; Kim et al. 2013) for the biosynthesis of chlorogenic acid. As an acyl-CoA-dependent transferase, HCT is capable of acylating a wide variety of acceptors, with some exhibiting broad substrate flexibility (Chiang et al. 2018; Eudes et al. 2016). Crystal structures of HCTs from different plants have been determined for both the apo-form and complexed structure with diverse substrates allowing determination of active sites. For example, the apo-form and ternary complex with p-coumaroyl-CoA and shikimate of SbHCT from Sorghum bicolor (L.) Moench revealed the catalytic mechanism of HCT (Walker et al. 2013). Structures of AtHCT from Arabidopsis thaliana (L.), CbHCT from Coleus blumei Benth, CcHCT from Coffea canephora Pierre ex Froehn and SmHCT from Selaginella moellendorffii Hieron. have also been reported (Chiang et al. 2018; Lallemand et al. 2012; Levsh et al. 2016).

Similar to other key genes involved in monolignol biosynthesis, HCT is found as a gene family in many species in plant kingdom (Carocha et al. 2015; Ferreira et al. 2019; Ma et al. 2017; Raes et al. 2003; Zhang et al. 2018). The structural information and the proposed active sites of HCT, can help us to distinguish bona fide HCT utilizing shikimate as an acceptor and involved in monolignol biosynthesis in plants. In this study, we used genome-wide screening to identify $10 \mathrm{HCT}$ homologs in Populus trichocarpa. Further motif and active site analysis showed the divergence of PoptrHCTs. Expression profiles and co-expression network analysis identified PoptrHCT1 and 2 as the lignin-related HCTs. Finally, we cloned and characterized the catalytic 
70

71

72

73

activity of PtoHCT1 from Populus tomentosa in vitro, which generated caffeoyl shikimate. PtoHCT1 could be used as the target gene for genetic modification to alter lignin content and composition.

\section{Materials and Methods}

\section{Materials}

Leaves of six--year-old Populus tomentosa 741 were collected from Hebei, China (Hu et al. 2019; Tian et al. 2013). Samples were immediately frozen in liquid nitrogen and then stored at $-80{ }^{\circ} \mathrm{C}$ until use.

\section{Genome-wide identification of HCT gene family members}

To identify the HCT sequences in Populus, we first built a hidden Markov model (HMM) using reported HCT and HQT sequences. HMMsearch using Hmmer 3.0 software against the proteome data of Populus trichocarpa was performed based on the HMM model (Eddy 2010). The cutoff for PoptrHCT homolog screening was an E-value $(<\mathrm{E}-100)$ of both the domain and full sequence and scores of full sequences $(>400)$ (Supplementary Table S1). The stable gene ID and symbols for HCTs reported in a previous study were also marked in Supplementary Table S1 Table S2. The sequences used for building the HMM model are shown in Supplementary TableS2.

\section{Distribution of HCT genes and HCT orthologs on Populus chromosomes}

Ten candidate HCT and HCT orthologs were located on chromosomes in specific duplicated blocks which were determined based on the Populus genome and the WGDotplot in the PLAZA platform (Proost et al. 2009).

\section{HCT sequence alignment and phylogenetic analysis}

Alignment of PoptrHCTs and SbHCT, AtHCT were performed using DNAman 8.0 (Lynnon BioSoft) with default parameters. A phylogenetic tree was obtained using Mega 7.0 with the maximum-likelihood method (Kumar et al. 2016; Tamura et al. 2011). The phylogenetic tree was assessed by bootstrapping using 1000 bootstrap replicates and marked above nodes only if greater than 50. The JTT substitution model and G+I rates among sites model were selected as parameters for building the tree. The putative HCT sequences are listed in Supplementary Table S2.

\section{HCT expression profiles in $P$. trichocarpa and $P$. tomentosa}

We obtained gene expression profiles for various tissues in P. trichocarpa using the GEO database with the accession number GSE30507. In addition, RNA-seq dataset GSE78953 including the transcriptome of various 
97

monolignol biosynthesis related mutants in P. trichocarpa, was used for co-expression analysis to explore the functions of the PoptrHCT orthologs. We also examined the expression profiles of PtoHCT orthologs in P. tomentosa in different seasons (Spring, Summer, Fall and Winter) and organs or tissues ( roots, buds, phloem and xylem) using our microarray dataset (accession number: GSE56023 ) (Chao et al. 2014b). The corresponding PtoHCTs were identified using PoptrHCTs as queries by local blastn against the probe sequences database (Christiam et al. 2009) TBtools v0.6652 and Cytoscape 3.4 were used to visualize the HCT expression profile or co-expression network (Chen et al. 2018; Shannon et al. 2003) (Supplementary Table S3).

\section{Cloning and purification of recombinant HCT from $P$. tomentosa}

Isolation of RNA and cDNA synthesis have been described in a previous study (Chao et al. 2014a) .We cloned the homologous $H C T 1$ from $P$. tomentosa based on the sequence information from $P$. trichocarpa (GenBank accession number: KT021003). Primer pair used for PCR amplification of PtoHCT1 is as follow: forward, 5'-CGATAAATAGAGCATTAGCACGGGG-3'; and reverse, 5'-ATAG CCTCGGCTCATTCTTT3'. PCR products were purified and cloned into the pMD18-T vector (Takara Dalian), propagated in Escherichia coli DH5 $\alpha$ and inserts were confirmed by sequencing. PtoHCT1 was constructed with pET28a (Novagen) through a digestion-ligation way using restriction enzymes BamHI, HindIII and T4 ligation (Takara, Dalian). pET28a -PtoHCT1was then transformed into E.coli BL21(DE3). To induce expression, Isopropy- $\beta$-Dthiogalactoside (IPTG)was added to a final concentration of $0.8 \mathrm{mM}$ and incubation was continued at $28^{\circ} \mathrm{C}$ for four hours. Cells were collected by centrifugation at $4000 \mathrm{~g}$ and $4^{\circ} \mathrm{C}$ for $15 \mathrm{~min}$. The pellets were resuspended in lysis buffer $(50 \mathrm{mM} \mathrm{NaH} 2 \mathrm{PO}, 300 \mathrm{mM} \mathrm{NaCl}$ with $10 \mathrm{mM}$ imidazole, $\mathrm{pH} 8.0)$ and then disrupted by sonication. After centrifugation at $12,000 \mathrm{~g}$ and $4{ }^{\circ} \mathrm{C}$ for $30 \mathrm{~min}$, the lysates were mixed with pretreated $1 \mathrm{ml} \mathrm{Ni}$-NTA agarose (Qiagen Shanghai, China)) After washing using lysis buffer supplemented with 20mM imidazole, the His-tagged PtoHCT1was eluted with $100 \mathrm{mM}$ imidazole in lysis buffer.

\section{Catalytic activity of recombinant PtoHCT1}

Caffeoyl-CoA was chemically synthesized as reported (Chao et al. 2017). We determined the activity of recombinant PtoHCT1 by synthesis of caffeoyl shikimate using caffeoyl-CoA and shikimic acid as substrates.

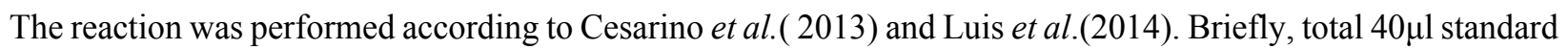
reaction mix contained $100 \mathrm{mM}$ Tris-HCl $\mathrm{pH}$ 7, 1mM DTT, $100 \mu \mathrm{M}$ caffeoyl-CoA, 100 $\mu \mathrm{M}$ shikimic acid and 
124

125

126

127

128

129

130

131

132

133

134

135

136

137

138

139

140

141

142

143

144

145

146

147

148

149

150

$10 \mu \mathrm{g}$ purified recombinant HCT protein. The reaction was initiated by adding the HCT proteins or the same amount of boiled protein as negative control. After Incubating at $30{ }^{\circ} \mathrm{C}$ for $30 \mathrm{~min}$, the reaction was terminated by boiling the samples for $5 \mathrm{~min}$. Flow for HPLC analysis was $0.1 \mathrm{~mL} / \mathrm{min}$ in solvent A (acetonitrile) and solvent $\mathrm{B}(0.01 \%$ formic acid in water). The gradient was $0 \% \mathrm{~A}$ to $35 \%$ in $\mathrm{B}$ for 0 to $24 \mathrm{~min}, 35 \% \mathrm{~A}$ in $\mathrm{B}$ to $100 \% \mathrm{~B}$ for 24 to $27 \mathrm{~min}, 100 \% \mathrm{~B}$ for 27 to $32 \mathrm{~min}, 100 \%$ A to $100 \% \mathrm{~B}$ for 32 to $35 \mathrm{~min}$, and $100 \% \mathrm{~B}$ for 35 to $55 \mathrm{~min}$. The parameters used for MS analysis was sheath gas (nitrogen) flow rate, 40 arb; aux/sweep gas (nitrogen) flow rate, 10 arb; spray voltage, $4.5 \mathrm{kV}$; capillary temperature, $320^{\circ} \mathrm{C}$. Optimized detailed parameters for dissociation of parent ions into product ions for each compound were provided in Supplementary Table S4.

\section{Structure modeling of PtoHCT1}

The crystal structure of AtHCT (accession number 5KJT) (Levsh et al. 2016) was obtained from the Protein Data Bank to build a homolog model for PtoHCT (https://www.rcsb.org). Molecular docking was performed using CDOCKER assembled in Discovery Studio 4.5. Visualization of the active sites and 3-D structures were generated by Discovery Studio 4.5.

\section{Results}

\section{Genome-wide identification and distribution of HCT orthologs in Populus}

Ten PoptrHCT homologs were found based on HMMsearch against the Populus genome (Supplementary Table S1). These $H C T$ candidate genes are located on six different chromosomes. Among the 10 PoptrHCT orthologs, PoptrHCT3, 4 and 5 were located on chromosome V, and PoptrHCT7, 8, 9, and 10, were on chromosome XVIII, representing two clusters respectively (Figure 2A). Tandem duplication is likely to be responsible for the formation of HCT homolog clusters. Ks (substitution per synonymous site) value distributions can be used for revealing whole genome duplication (WGD) events (Jiao et al. 2011; Tang et al. 2010). PoptrHCT1 and PoptrHCT2 formed a homolog duplicate pair with $K s$ value 0.2174 and were located at corresponding homologous duplicated blocks, as the result of whole genome duplication. The organization of the PoptrHCT orthologs indicates that both genome duplication and tandem duplication played roles in the formation of the HCT family.

\section{Alignment and phylogenetic analysis of HCT orthologs}

Putative protein sequences of PoptrHCT orthologs and crystal structures of two shikimate-specific HCTs 
151

152

153

154

155

156

157

(AtHCT and SbHCT) and LeHQT (Lycopersicon esculentum Mill.) were aligned. Characteristic of the BADH superfamily, two motifs HXXXD(G) and DFGWG were conserved in AtHCT, SbHCT and all PoptrHCT orthologs (except PoptrHCT6) (Figure 3) (D'Auria 2006). Based on previous studies of the structure of HCTs including site-directed mutagenesis, molecular docking and crystallographic analyses we summarized the active sites of HCTs (Table1) and marked these active sites in Figure 3. Active sites for the carbonyl group of the $p$ coumaroyl moiety binding and the catalysis related sites of LeHQT correspond with HCTs (red full circles) while divergence is obvious in terms of active sites for shikimate binding (red full stars). PoptrHCT1 and PoptrHCT2 showed conservation at these active sites and kept correspondence with AtHCT and SbHCT, while PoptrHCT3-10 showed poor conservation at these key sites. Thus while the ten candidate PoptrHCTs mostly belong to the BADH superfamily, only PoptrHCT1 and PoptrHCT2 appear to be associated with monolignol biosynthesis. Phylogenetic analysis showed PoptrHCT1 and PoptrHCT2 grouped with Group I HCTs, which transfer hydroxycinnamates to shikimate and have been implicated in monolignol biosynthesis, strongly suggesting this role for PoptrHCT1 and 2 as well (Figure 2B). Other PoptrHCTs (3-10) clustered with HQTs and other HCT-like as Group II and especially, PoptrHCT6 without DFGWG seem unlikely to be shikimatespecific transferases involved in monolignol biosynthesis. Thus these Group II. PoptrHCTs might have different catalytic activity (e.g., utilize acceptors other than shikimic acid) and are likely to play different roles in plants.

\section{Expression analysis of HCT homolog genes}

Based on the microarray analysis of seven different tissues and organs in P. trichocarpa, PoptrHCT1 and 2 showed expression preference in developing xylem (DX) and mature xylem (MX). PoptrHCT1 showed high expression levels in all detected tissues and organs. PoptrHCT9 and 10 showed expression preference in developing tissues including developing phloem, developing xylem, cambium (C) and shoots and leaf primordium (SLp) (Figure 4A). Co-expression network analysis shows that PoptrHCT1 and 2 have significant correlations with genes involved in lignin biosynthesis (Figure 4B). Monolignol biosynthesis related transcription factors also showed co-expression with PoptrHCT1 and 2. The similar expression patterns were also found in P. tomentosa Carr. (Pto). PtoHCT1 has high expression levels in almost all tissues and organs yearround while PtoHCT9 and 10 showed preference in buds and phloem especially in winter, which indicates that these two genes could be involved in development of dormancy and response to cold stress. The differential 
178

179

180

181

182

183

expression of the PoptrHCT orthologs further supports that HCT1 plays a major role in monolignol biosynthesis.

\section{Catalytic activity and structure comparison of PtoHCT1}

PtoHCT1 protein expressed in E. coli was purified for enzymatic assays. We monitored PtoHCT1 reactions using HPLC-MS and found that PtoHCT1can utilize caffeoyl-CoA and shikimic acid (Figure 5). After the initiation of the reaction by adding PtoHCT1, the accumulation of caffeoyl shikimate and decrease of caffeoyl-CoA and shikimic acid were observed within 2 minutes. We built a homology model for PtoHCT1 to explore the structure of PtoHCT1 using the crystal structure of AtHCT (accession number 5KJT) as template. The main-chain rootmean-square deviation (RMSD) is $0.224 \AA$ indicating the high structure similarity of PtoHCT1 and AtHCT

(Figure 6A). According to the summarized active sites (Table 1), we found these conserved sites around the catalytic cleft, and then we successfully docked PtoHCT1 with the substrate caffeoyl-CoA, which further provided the structural evidence for PtoHCT1 catalyzing caffeoyl-CoA (Figure 6B).

\section{Discussion}

HCT regulates the flux at a key point in monolignol biosynthesis and has been studied in many plants (Hoffmann et al. 2004; Hoffmann et al. 2003; Shadle et al. 2007; Sun et al. 2018; Wagner et al. 2007). HCT also exists as a gene family in the land plant kingdom similar to other key genes involved in monolignol biosynthesis. We focused on the HCT gene family in this study and provided a systematic analysis of the HCT genes in poplar, and identified two lignin-related HCTs (HCTland HCT2), which exist as a homolog pair located at a duplication block on chromosome I and III respectively (Figure 2A). Ks analysis indicated that the HCT gene pair (PoptrHCT1 and PoptrHCT2) resulted from a recent genome duplication (Ks value 0.2174). Two HCT homolog clusters resulting from tandem duplication were also identified. Both genome duplication and tandem duplication provide raw genetic material for neo-function as a driving force in plant evolution and are responsible for the expansion of HCT orthologs (Zhang 2003).

Based on our systematic analysis, PoptrHCT1 and 2 are involved in monolignol biosynthesis. Both PoptrHCT1 and 2 showed expression preference in xylem and co-expression with other monolignol related genes. HCT1 (PoptrHCT1 and PtoHCT1) had high expression levels in different tissues. PtoHCT1 showed catalytic activity for caffeoyl-CoA and shikimic acid. These results further validate $H C T 1$ as the dominant HCT in monolignol biosynthesis, while HCT9 and 10 (PoptrHCT9, 10 and PtoHCT9, 10) showed expression 
205

206

207

208

209

210

211

212

213

214

215

216

217

218

219

220

221

222

223

224

225

226

227

228

229

230

231

preference in developing tissues. PtoHCT9 and 10 were active in winter, which suggested a function in plant development and response to cold stress. PtoHCT1 utilizes caffeoyl-CoA and shikimic acid to generate caffeoyl shikimate which can be used as substrate for CSE, a new annotated enzyme involved in monolignol biosynthesis (Ha et al. 2016; Saleme et al. 2017; Vanholme et al. 2013).

\section{Conclusion}

In summary, we identified ten HCT homologs and proposed the important roles of both genome duplication and tandem duplication in the expansion of HCT orthologs in Populous. Two HCTs likely involved in monolignol biosynthesis in Populus were identified based on phylogenetic analysis and expression profile analysis. Enzymatic assay of PtoHCT1 showed that PtoHCT1 was able to synthetize caffeoyl shikimate using caffeoyl-CoA and shikimic acid as substrates. In addition, other PoptrHCT orthologs showed divergence in reported active sites and different expression pattern. HCT9 and 10 (PoptrHCT9, 10 and PtoHCT9, 10) showed preferential expression in developing tissues and were active in winter. Further studies should help to reveal the functions of the other HCT orthologs.

Supplementary Table S1 Genome-wide screening PoptrHCT genes based on HMM models; Supplementary Table S2 Putative HCTs used for phylogenetic analysis;

Supplementary Table S3 Expression profiles and co-expression for HCT homologs.

Supplementary Table S4 Conditions used for HPLC-MS to identify caffeic acid, shikimate and caffeoyl shikimate

Supplementary File 5: Sequences used for alignment analysis.

\section{Acknowledgements}

This work was jointly supported by the Beijing Higher Education Young Elite Teacher Project [YETP0755 granted to Dr. Ying Gai], the National Natural Science Foundation [NSF 31300498 to Ying Gai].

\section{Author Contributions}

Ying Gai and Xiang-Ning Jiang guided the work and provided advice; Qi Qi, Shuang Li and Nan Chao performed the experiments; Brent Ruan and Nan Chao read and revised the draft; Nan Chao analyzed the data and wrote the paper.

\section{Conflict of Interest}


232 The authors declare that the research was conducted in the absence of any commercial or financial

233 relationships that could be construed as a potential conflict of interest.

\section{Reference}

235

236

237

238

239

240

241

242

243

244

245

246

247

248

249

250

251

252

253

254

255

256

257

258

259

260

261

262

263

264

265

266

267

268

269

270

271

Boerjan W, Ralph J, and Baucher M. 2003. Lignin biosynthesis. Annual Review of Plant Biology 54:519-546. 10.1146/annurev.arplant.54.031902.134938

Carocha V, Soler M, Hefer C, Cassan-Wang H, Fevereiro P, Myburg AA, Paiva JA, and Grima-Pettenati J. 2015. Genome-wide analysis of the lignin toolbox of Eucalyptus grandis. New Phytologist 206:1297-1313. 10.1111/nph.13313

Chao N, Li N, Qi Q, Li S, Lv T, Jiang X-N, and Gai Y. 2017. Characterization of the cinnamoyl-CoA reductase (CCR) gene family in Populus tomentosa reveals the enzymatic active sites and evolution of CCR. Planta 245:61-75.

Chao N, Liu S-X, Liu B-M, Li N, Jiang X-N, and Gai Y. 2014a. Molecular cloning and functional analysis of nine cinnamyl alcohol dehydrogenase family members in Populus tomentosa. Planta 240:1097-1112.

Chao N, Liu SX, Liu BM, Li N, Jiang XN, and Gai Y. 2014b. Molecular cloning and functional analysis of nine cinnamyl alcohol dehydrogenase family members in Populus tomentosa. Planta 240:1097-1112. 10.1007/s00425014-2128-9

Chen C, Xia R, Chen H, and He Y. 2018. TBtools, a Toolkit for Biologists integrating various HTS-data handling tools with a user-friendly interface.

Chiang YC, Levsh O, Lam CK, Weng JK, and Wang Y. 2018. Structural and dynamic basis of substrate permissiveness in hydroxycinnamoyltransferase (HCT). PLoS Comput Biol 14:e1006511. 10.1371/journal.pcbi.1006511

Christiam, Camacho, George, Coulouris, Vahram, Avagyan, Ning, Ma, Jason, and Papadopoulos. 2009. BLAST+: architecture and applications. BMC Bioinformatics.

D’Auria JC. 2006. Acyltransferases in plants: a good time to be BAHD. Current Opinion in Plant Biology 9:331-340.

Eddy S. 2010. HMMER3: a new generation of sequence homology search software. URL: http://hmmer janelia Org.

Eudes A, Pereira JH, Yogiswara S, Wang G, Benites VT, Baidoo EE, Lee TS, Adams PD, Keasling JD, and Loqué D. 2016. Exploiting the Substrate Promiscuity of Hydroxycinnamoyl-CoA: Shikimate Hydroxycinnamoyl Transferase to Reduce Lignin. Plant and Cell Physiology 57:568-579.

Ferreira SS, Simoes MS, Carvalho GG, De Lima LGA, Svartman RMDA, and Cesarino I. 2019. The lignin toolbox of the model grass Setaria viridis. Plant Molecular Biology:1-21.

Ha CM, Escamilla-Trevino L, Yarce JC, Kim H, Ralph J, Chen F, and Dixon RA. 2016. An essential role of caffeoyl shikimate esterase in monolignol biosynthesis in Medicago truncatula. Plant Journal 86:363-375. 10.1111/tpj.13177

Hoffmann L, Besseau S, Geoffroy P, Ritzenthaler C, Meyer D, Lapierre C, Pollet B, and Legrand M. 2004. Silencing of hydroxycinnamoyl-coenzyme A shikimate/quinate hydroxycinnamoyltransferase affects phenylpropanoid biosynthesis. The Plant Cell 16:1446-1465.

Hoffmann L, Maury S, Martz F, Geoffroy P, and Legrand M. 2003. Purification, cloning, and properties of an acyltransferase controlling shikimate and quinate ester intermediates in phenylpropanoid metabolism. Journal of Biological Chemistry 278:95-103.

Hu JQ, Qi Q, Zhao YL, Tian XM, Lu H, Gai Y, and Jiang XN. 2019. Unraveling the impact of Pto4CL1 regulation on the cell wall components and wood properties of perennial transgenic Populus tomentosa. Plant Physiology and Biochemistry 139:672-680. 10.1016/j.plaphy.2019.03.035

Peer) reviewing PDF | (2020:02:45737:5:0:NEW 12 Dec 2020) 
272

273

274

275

276

277

278

279

280

281

282

283

284

285

286

287

288

289

290

291

292

293

294

295

296

297

298

299

300

301

302

303

304

305

306

307

308

309

310

311

312
Jiao Y, Wickett NJ, Ayyampalayam S, Chanderbali AS, Landherr L, Ralph PE, Tomsho LP, Hu Y, Liang H, and Soltis PS. 2011. Ancestral polyploidy in seed plants and angiosperms. Nature 473:97-100.

Karkonen A, and Koutaniemi S. 2010. Lignin biosynthesis studies in plant tissue cultures. J Integr Plant Biol 52:176185. 10.1111/j.1744-7909.2010.00913.x

JIPB913 [pii]

Kim YB, Thwe AA, Kim YJ, Li X, Kim HH, Park PB, Suzuki T, Kim S-J, and Park SU. 2013. Characterization of Genes for a Putative Hydroxycinnamoyl-coenzyme A Quinate Transferase and p-Coumarate 3' -Hydroxylase and Chlorogenic Acid Accumulation in Tartary Buckwheat. Journal of Agricultural and Food Chemistry 61:41204126.

Kumar S, Stecher G, and Tamura K. 2016. MEGA7: Molecular Evolutionary Genetics Analysis Version 7.0 for Bigger Datasets. Molecular Biology \& Evolution 33:1870.

Lallemand LA, Zubieta C, Lee SG, Wang Y, Acajjaoui S, Timmins J, Mcsweeney S, Jez JM, Mccarthy J, and Mccarthy AA. 2012. A STRUCTURAL BASIS FOR THE BIOSYNTHESIS OF THE MAJOR CHLOROGENIC ACIDS FOUND IN COFFEE. Plant Physiology 160:249-260.

Lepelley M, Cheminade G, Tremillon N, Simkin A, Caillet V, and McCarthy J. 2007. Chlorogenic acid synthesis in coffee: An analysis of CGA content and real-time RT-PCR expression of HCT, HQT, C3H1, and CCOAOMT1 genes during grain development in C. canephora. Plant Science 172:978-996.

Levsh O, Chiang Y, Tung CF, Noel JP, Wang Y, and Weng J. 2016. Dynamic Conformational States Dictate Selectivity toward the Native Substrate in a Substrate-Permissive Acyltransferase. Biochemistry 55:6314-6326.

Li X, Bonawitz ND, Weng JK, and Chapple C. 2010. The growth reduction associated with repressed lignin biosynthesis in Arabidopsis thaliana is independent of flavonoids. Plant Cell 22:1620-1632. 10.1105/tpc.110.074161

Ma C, Zhang H, Li J, Tao S, Qiao X, Korban SS, Zhang S, and Wu J. 2017. Genome-wide analysis and characterization of molecular evolution of the HCT gene family in pear (Pyrus bretschneideri). Plant Systematics and Evolution 303:71-90.

Maeda HA. 2016. Lignin biosynthesis: Tyrosine shortcut in grasses. Nat Plants 2:16080. 10.1038/nplants.2016.80

Poovaiah CR, Nageswara - Rao M, Soneji JR, Baxter HL, and Stewart CN. 2014. Altered lignin biosynthesis using biotechnology to improve lignocellulosic biofuel feedstocks. Plant Biotechnology Journal.

Proost S, Van Bel M, Sterck L, Billiau K, Van Parys T, Van de Peer Y, and Vandepoele K. 2009. PLAZA: a comparative genomics resource to study gene and genome evolution in plants. Plant Cell 21:3718-3731. 10.1105/tpc.109.071506

Raes J, Rohde A, Christensen JH, De Peer YV, and Boerjan W. 2003. Genome-Wide Characterization of the Lignification Toolbox in Arabidopsis. Plant Physiology 133:1051-1071.

Ralph J, Lapierre C, and Boerjan W. 2019. Lignin structure and its engineering. Current Opinion in Biotechnology 56:240-249. 10.1016/j.copbio.2019.02.019

Saleme MLS, Cesarino I, Vargas L, Kim H, Vanholme R, Goeminne G, Van Acker R, Fonseca FCA, Pallidis A, Voorend W, Junior JN, Padmakshan D, Van Doorsselaere J, Ralph J, and Boerjan W. 2017. Silencing CAFFEOYL SHIKIMATE ESTERASE Affects Lignification and Improves Saccharification in Poplar. Plant Physiology 175:1040-1057. 10.1104/pp.17.00920

Shadle G, Chen F, Reddy MS, Jackson L, Nakashima J, and Dixon RA. 2007. Down-regulation of hydroxycinnamoyl CoA: shikimate hydroxycinnamoyl transferase in transgenic alfalfa affects lignification, development and forage quality. Phytochemistry 68:1521-1529.

Peer] reviewing PDF | (2020:02:45737:5:0:NEW 12 Dec 2020) 
313

314

315

316

317

318

319

320

321

322

323

324

325

326

327

328

329

330

331

332

333

334

335

336

337

338

339

340

341

342

343

344

345

346

347

348

349

350

351

352

353

Shannon P, Markiel A, Ozier O, Baliga NS, Wang JT, Ramage D, Amin N, Schwikowski B, and Ideker T. 2003. Cytoscape: A Software Environment for Integrated Models of Biomolecular Interaction Networks. Genome Research 13:2498-2504.

Sun $\mathrm{CH}$, Yang $\mathrm{CY}$, and Tzen JTC. 2018. Molecular Identification and Characterization of Hydroxycinnamoyl Transferase in Tea Plants (Camellia sinensis L.). Int J Mol Sci 19. 10.3390/ijms19123938

Tamura K, Peterson D, Peterson N, Stecher G, Nei M, and Kumar S. 2011. MEGA5: molecular evolutionary genetics analysis using maximum likelihood, evolutionary distance, and maximum parsimony methods. Molecular biology and evolution 28:2731-2739.

Tang H, Bowers JE, Wang X, and Paterson AH. 2010. Angiosperm genome comparisons reveal early polyploidy in the monocot lineage. Proceedings of the National Academy of Sciences 107:472-477.

Tang W, and Tang AY. 2014. Transgenic woody plants for biofuel. Journal of Forestry Research 25:225-236.

Tian X, Xie J, Zhao Y, Lu H, Liu S, Qu L, Li J, Gai Y, and Jiang X. 2013. Sense-, antisense- and RNAi-4CL1 regulate soluble phenolic acids, cell wall components and growth in transgenic Populus tomentosa Carr. Plant Physiology and Biochemistry 65:111-119. 10.1016/j.plaphy.2013.01.010

Vanholme R, Cesarino I, Rataj K, Xiao Y, Sundin L, Goeminne G, Kim H, Cross J, Morreel K, and Araujo P. 2013. Caffeoyl shikimate esterase (CSE) is an enzyme in the lignin biosynthetic pathway in Arabidopsis. Science 341:11031106.

Vanholme R, De Meester B, Ralph J, and Boerjan W. 2019. Lignin biosynthesis and its integration into metabolism. Current Opinion in Biotechnology 56:230-239. 10.1016/j.copbio.2019.02.018

Vanholme R, Demedts B, Morreel K, Ralph J, and Boerjan W. 2010. Lignin biosynthesis and structure. Plant Physiology 153:895-905. 10.1104/pp.110.155119

pp.110.155119 [pii]

Vargas L, Cesarino I, Vanholme R, Voorend W, de Lyra Soriano Saleme M, Morreel K, and Boerjan W. 2016. Improving total saccharification yield of Arabidopsis plants by vessel-specific complementation of caffeoyl shikimate esterase (cse) mutants. Biotechnol Biofuels 9:139. 10.1186/s13068-016-0551-9

Wagner A, Ralph J, Akiyama T, Flint H, Phillips L, Torr K, Nanayakkara B, and Te Kiri L. 2007. Exploring lignification in conifers by silencing hydroxycinnamoyl-CoA: shikimate hydroxycinnamoyltransferase in Pinus radiata. Proceedings of the National Academy of Sciences 104:11856-11861.

Walker AM, Hayes RP, Youn B, Vermerris W, Sattler SE, and Kang C. 2013. Elucidation of the structure and reaction mechanism of sorghum hydroxycinnamoyltransferase and its structural relationship to other coenzyme Adependent transferases and synthases. Plant Physiology 162:640-651.

Wang JP, Matthews ML, Naik PP, Williams CM, Ducoste JJ, Sederoff RR, and Chiang VL. 2019. Flux modeling for monolignol biosynthesis. Current Opinion in Biotechnology 56:187-192. 10.1016/j.copbio.2018.12.003

Wang JP, Naik PP, Chen HC, Shi R, Lin CY, Liu J, Shuford CM, Li Q, Sun YH, Tunlaya-Anukit S, Williams CM, Muddiman DC, Ducoste JJ, Sederoff RR, and Chiang VL. 2014. Complete proteomic-based enzyme reaction and inhibition kinetics reveal how monolignol biosynthetic enzyme families affect metabolic flux and lignin in Populus trichocarpa. Plant Cell 26:894-914. 10.1105/tpc.113.120881

Xu P, and Li L. 2016. Monolignol Biosynthesis and Regulation in Grasses. Recent Advances in Polyphenol Research 5:108.

Zhang J. 2003. Evolution by gene duplication: an update. Trends in Ecology and Evolution 18:292-298.

Zhang J, Yang Y, Zheng K, Xie M, Feng K, Jawdy SS, Gunter LE, Ranjan P, Singan VR, Engle N, Lindquist E, Barry K,

Peer) reviewing PDF | (2020:02:45737:5:0:NEW 12 Dec 2020) 
354

355

356

357

358

359

360

361
Schmutz J, Zhao N, Tschaplinski TJ, LeBoldus J, Tuskan GA, Chen JG, and Muchero W. 2018. Genome-wide association studies and expression-based quantitative trait loci analyses reveal roles of HCT2 in caffeoylquinic acid biosynthesis and its regulation by defense-responsive transcription factors in Populus. New Phytologist 220:502-516. 10.1111/nph.15297

Zhao Q. 2016. Lignification: flexibility, biosynthesis and regulation. Trends in Plant Science 21:713-721.

Zhong R, Cui D, and Ye ZH. 2019. Secondary cell wall biosynthesis. New Phytologist 221:1703-1723. 10.1111/nph.15537 
Figure 1

Schematic diagram of reaction catalyzed by HCT in monolignol biosynthesis pathway $R=$ Shikimate. 4CL, 4-coumarate-CoA ligase; $C 3 H$, p-coumarate 3-hydroxylase; $H C T$, Hydroxycinnamoyl-CoA: shikimate hydroxycinnamoyl transferase. Compounds in red shadow are precursors for $H$ units and in green shadow are for $G$ and $S$ units.<smiles>O=C(O)/C=C/c1ccc(O)cc1</smiles>

$p$-Coumaric acid

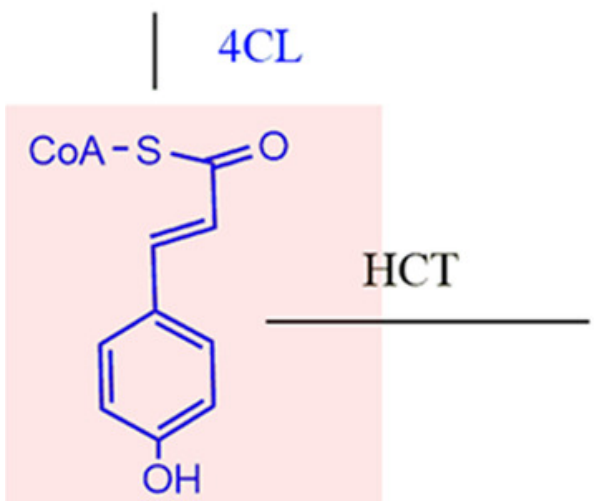

$p$-Coumaroyl-CoA $p$-Coumaroyl shikimic acid Caffeoyl shikimic acid
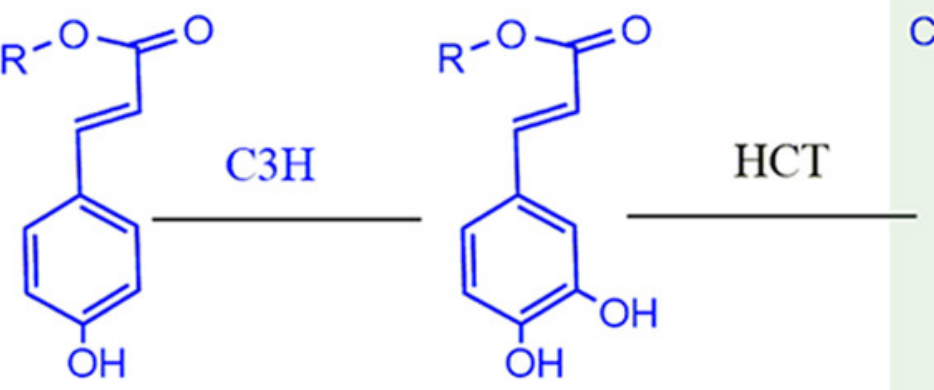

Caffeoyl-CoA 


\section{Figure 2}

PoptrHCT orthologs organization and phylogenetic analysis

(A). Organization of HCT orthologs on Populus chromosomes. Regions that are assumed to correspond to homologous genome blocks are shaded gray and connected by lines. The position of genes is indicated with an arrowhead. (B). Phylogenetic analysis of HCT homologs from Populus trichocarpa and other plant species. The PoptrHCT1 and 2 were marked with full black triangle. Two groups for HCT orthologs were shown and HCTs in Group I are likely to transfer hydroxycinnamates to shikimate and have been implicated in monolignol biosynthesis. The scale bar indicates 0.5 amino acid substitutions per site in given length. The accession numbers of sequences used are as followed: Arabidopsis thialiana AtHCT (AT5G48930), AtHCTlike (AT4G29250); Amborella trichopoda AtrHCT1 (ATR_00137G00320), AtrHCT2 (ATR_00727G00010); Cynara cardunculus CcaHCT (DQ104740), CcaHQT (ABK79690); Lycopersicon esculentum LeHQT ( AJ582652); Larix kaempferi LkaHCT (AHA44839); Nicotiana tabacum NtaHCT (Q8GSM7), NtaHQT(CAE46932); Picea lauca PgIHCT (CZ001061061); Populus trichocarpa PoptrHCT1 (PT01G04290), PoptrHCT2 (PT03G18390), PoptrHCT3 (PT05G02800), PoptrHCT4 (PT05G02810), PoptrHCT5 (PT05G02840), PoptrHCT6 (PT18G03270), PoptrHCT7 (PT18G10470), PoptrHCT8 (PT18G10480), PoptrHCT9 (PT18G10540), PoptrHCT10 (PT18G10550); Physcomitrella patens PpHCT1 (PP00022G00830); Panicum virgatum PviHCT1a (JX845714), PviHCT2 (KC696573); Sorghum bicolor SbHCT (XP_002452435.1)." 

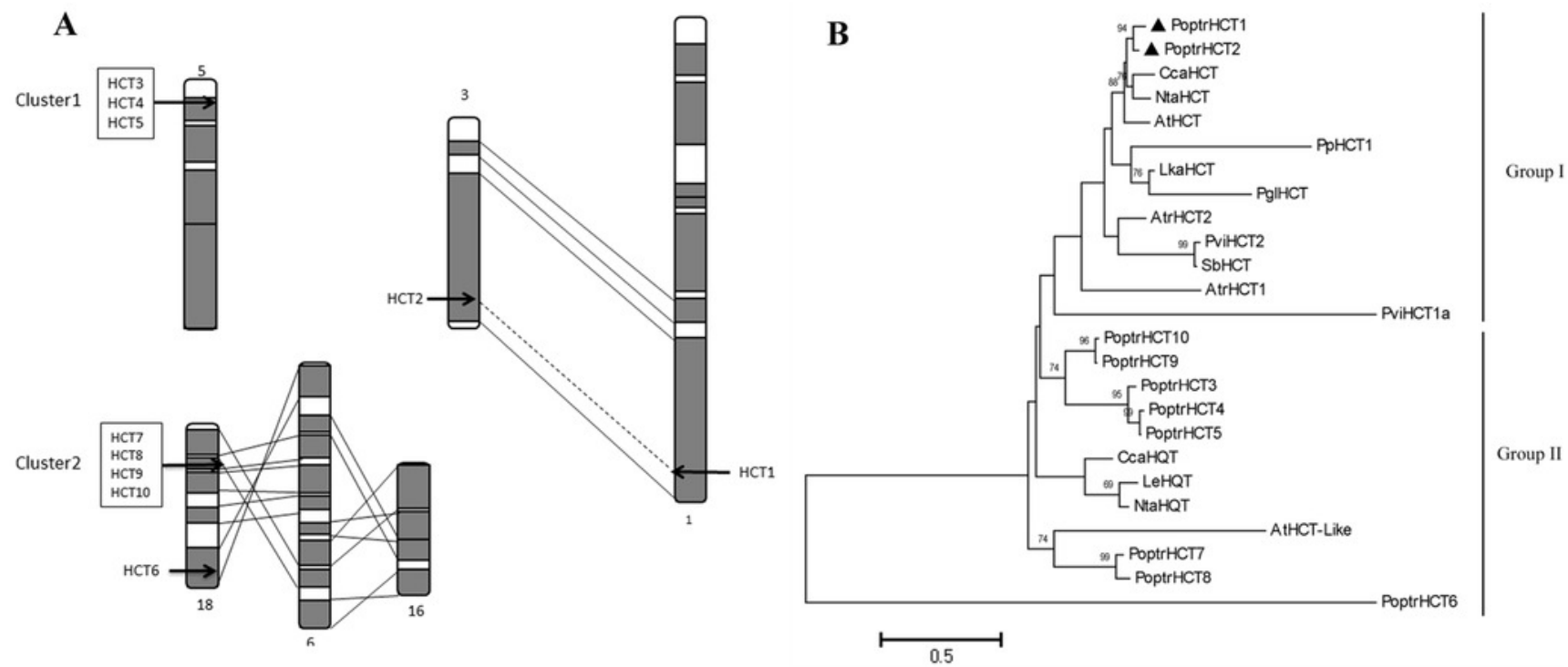


\section{Figure 3}

Alignment of PoptrHCT and PoptrHCT orthologs compared to shikimate-specific HCTs from Arabidopsis and sorghum and HQT from tomato.

Red full stars indicate shikimate binding sites, red full circles indicate carbonyl group of $p$ coumaroyl moiety binding sites, purple full triangle indicate carbonyl group of shikimate moiety binding sites and the blue full circles indicate sites involved in catalysis. Accessions are as in Figure 2. Detailed references are also available in Table 1 
SbHCT

AtHCT

LeHQT

PoptrHCT1

PoptrHCT2

PoptrHCT3

PoptrHCT 4

PoptrHCT5

PoptrHCT 6

PoptrHCT7

PoptrHCT8

PoptrHCT9

PoptrHCT10

SbHCT

AtHCT

LeHOT

PoptrHCT 1

PoptrHCT2

PoptrHCT3

PoptrHCT 4

PoptrHCT5

PoptrHCT 6

PoptrHCT 7

PoptrHCT

PoptrHCT 9

PoptrHCT10

SbHCT

AtHCT

LeHQT

PoptrHCT1

PoptrHCT2

PoptrHCT3

PoptrHCT 4

PoptrHCT 5

PoptrHCT 6

PoptrHCT7

PoptrHCT8

PoptrHCT 9

PoptrHCT10

SbHCT

AtHCT

LeHQT

PoptrHCT1

PoptrHCT2

PoptrHCT3

PoptrHCT 4

PoptrHCT5

PoptrHCT 6

PoptrHCT 7

PoptrHCT8

PoptrHCT9

PoptrHCT10

SbHCT

AtHCT

LeHOT

PoptrHCT 1

PoptrHCT2

PoptrHCT3

PoptrHCT 4

PoptrHCT5

PoptrHCT 6

PoptrHCT 7

PoptrHCT8

PoptrHCT9

PoptrHCT10
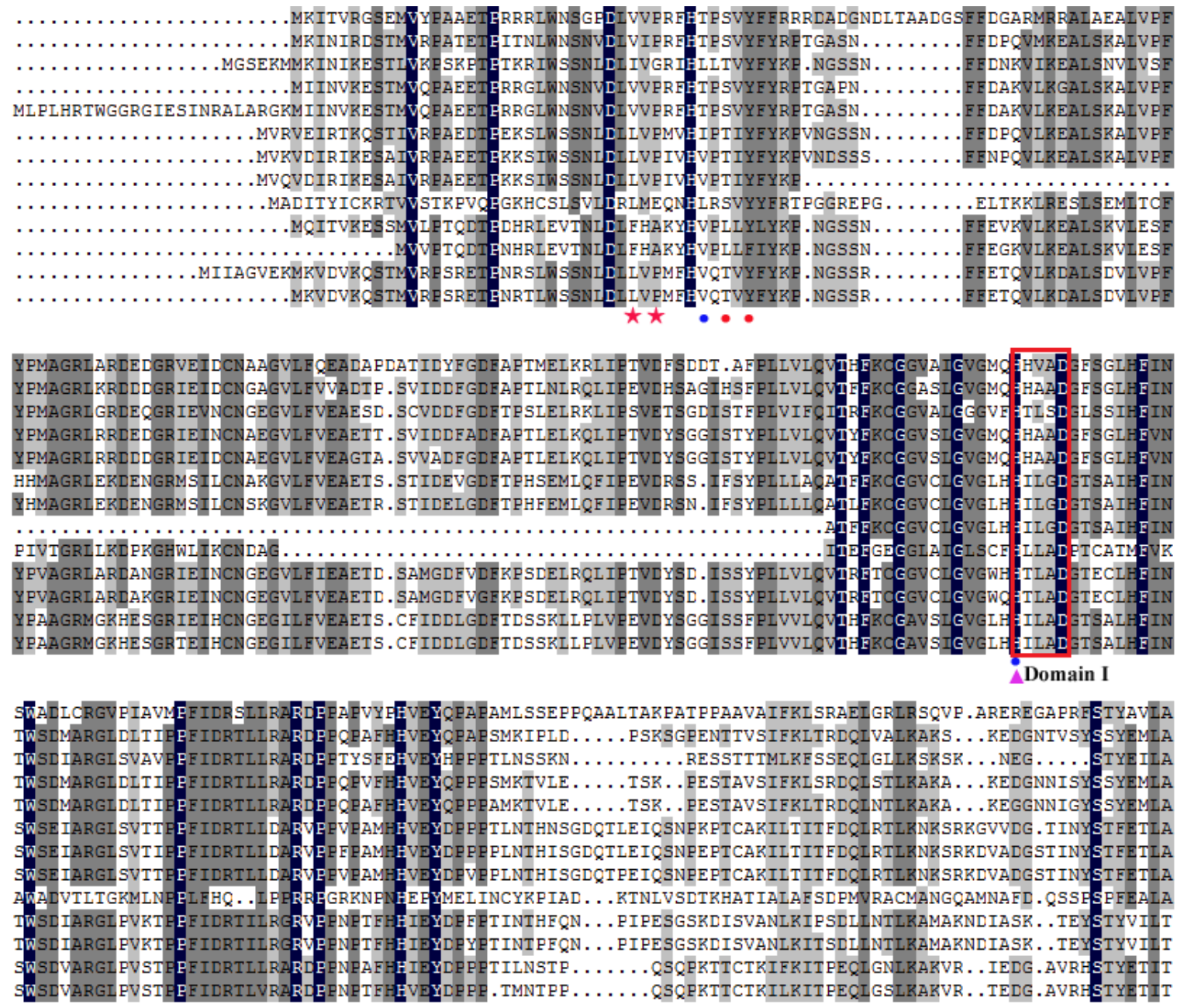

CEAEAMLSSEPPCAALTARPATPPAAVAIFRISRAEI GR LRSCVP. AREREGARR FSTYAVIA

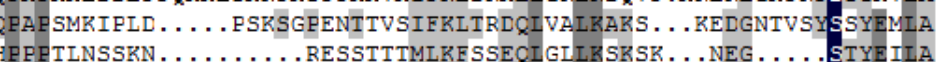

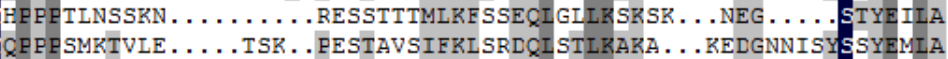
QPPEAMKTVLE ......TSK...PESTAVSIFRLTRDQINTLRARA .... KEGGNNIGYSSYEMIA DPPETLNTHNSGDQTLEIQSNPKPTCAKIITITFDQIRTLKNRSRKGVVDG .T INYSTFETLA YDFP PLNTHISGDQTLE IQSNPE PTCAKILTITFDQIRTLRNRSRKDVADGST INYST FETLA YDEVEPLNTHISGDQT PE IQSNEE PTCARILTIT FDQIRTLRNRSRKDVADGST INYST FETLA YMEL INCYRPIAD ... KTNLVSDTKHAT IALAF SDPMV RACMANGQAMNAFD. QSSESPFEAIA YDFF $T$ TINTHF $Q N$. . . PIPESGSKDISVANLRIPSDIINTLRAMAKNDIASK. . TEYSTYVIIT
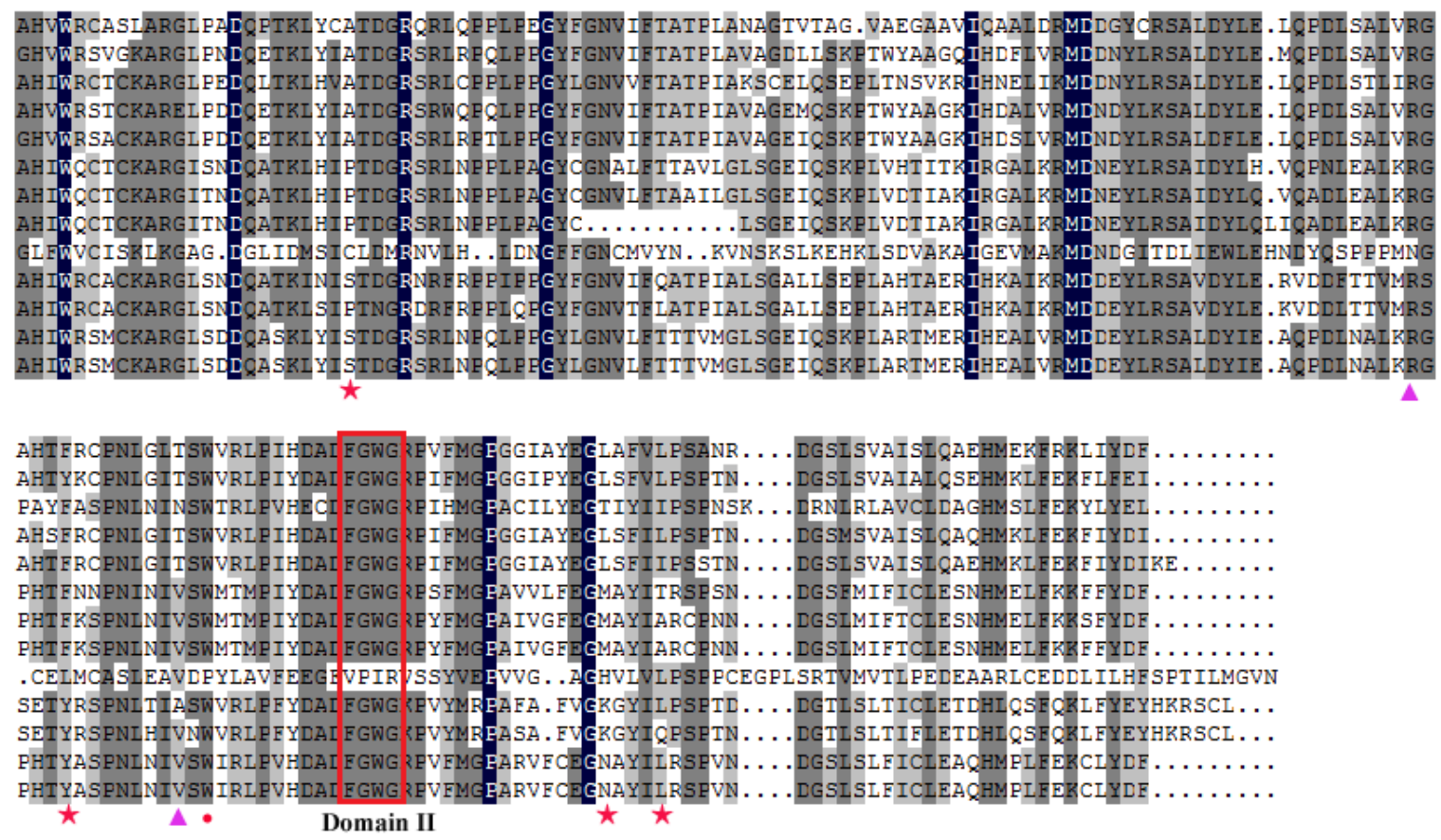


\section{Figure 4}

Expression profile and co-expression network of HCT orthologs in poplar.

(A). Expression profile of HCT orthologs in Populus. Tissues or specific parts of plants are indicated with the respective abbreviations: WS, whole stems; BM, Bark and mature phloem; C, cambium; DP, developing phloem; DX, developing xylem; ML, mature leaf; SLp, shoot and leaf primordium. (B) Expression profile of HCT orthologs in P. tomentosa. (C) Co-expression network of PoptrHCT orthologs with identified genes involved in lignin biosynthesis. The support information is available in Table S3. Only nodes with Pearson correlation coefficients $>0.9$ were shown and considered as close co-expression.
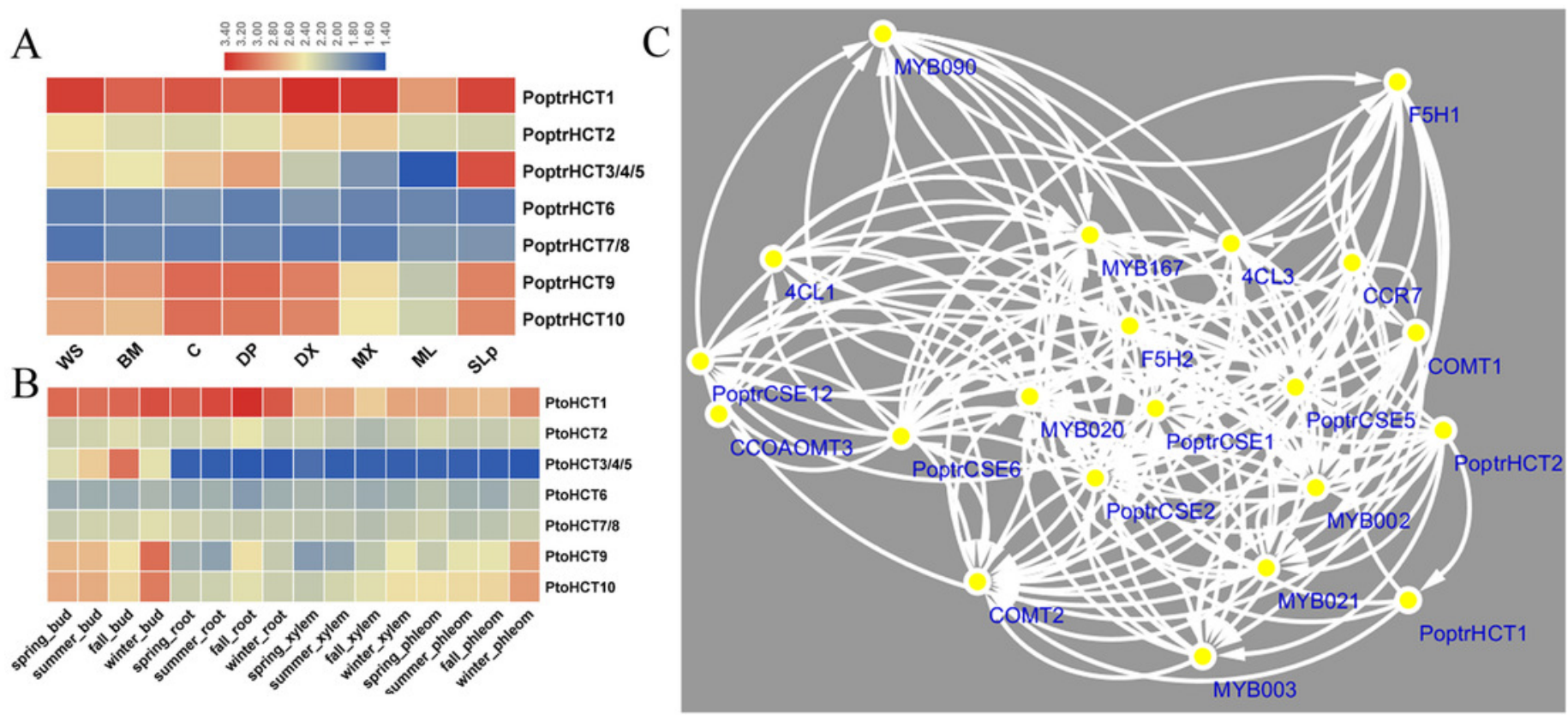
Figure 5

PtoHCT1 catalyzes enzymatic synthesis of caffeoyl shikimate.

$L C$ separation of reactions with MS detection (selected ion signals) (A) at initiation of the reaction (B) after 80 s or (C) after 120s 


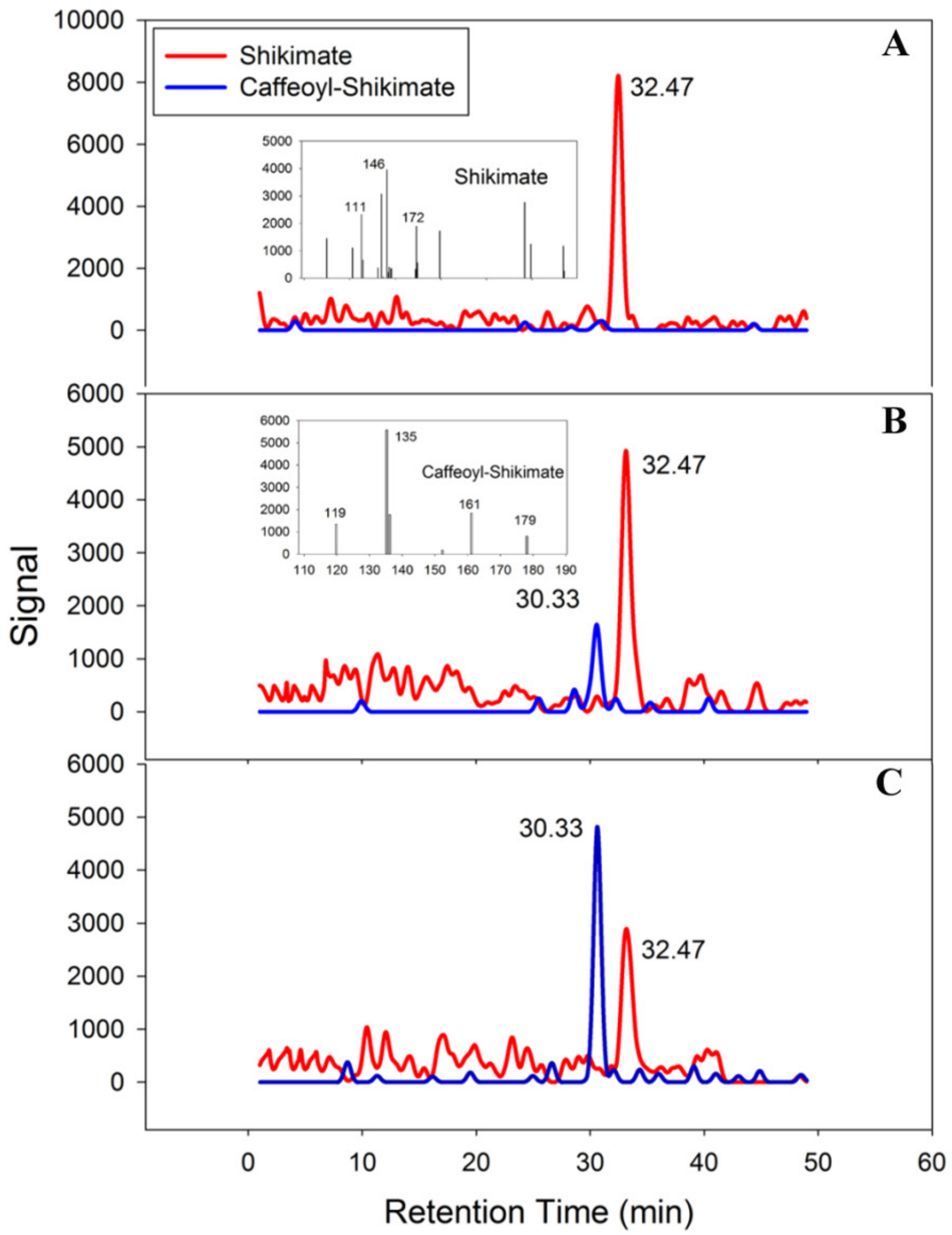


Figure 6

The structure of PtoHCT1 and docking with caffeoyl-CoA

(A) structure alignment of AtHCT (green) and PtoHCT (purple). (B) PtoHCT docked with caffeoyl-CoA. Blue ligand is caffeoyl-COA and active sites are labeled.

$\mathbf{A}$

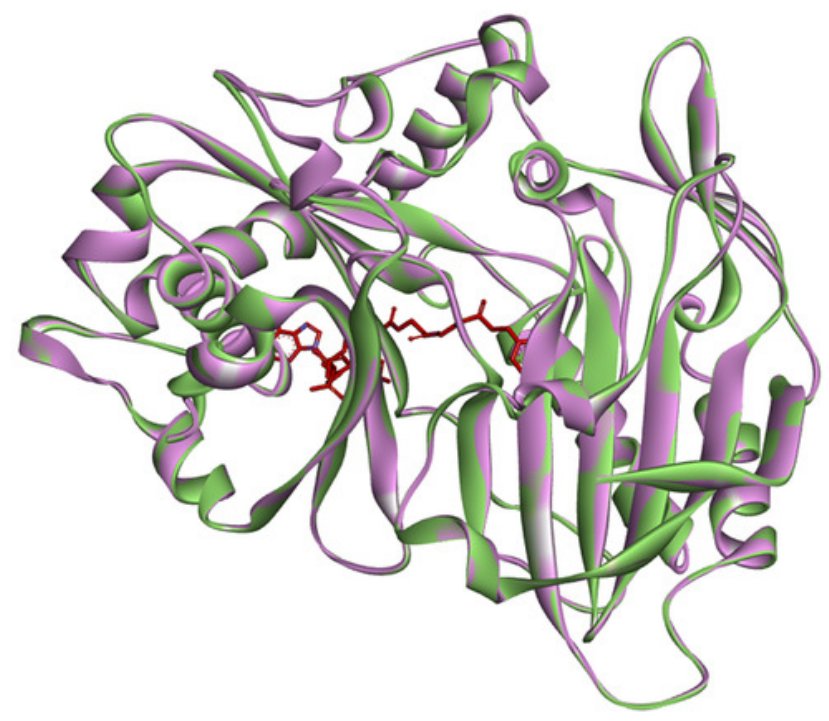

B

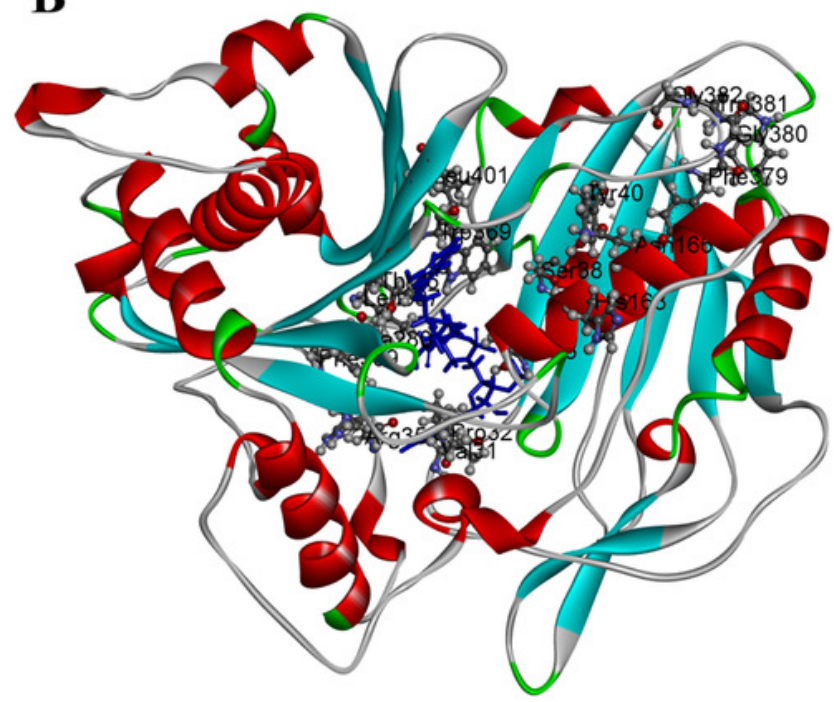




\section{Table 1 (on next page)}

Summary of active sites of HCTs 
1

\begin{tabular}{cccc}
\hline Position & Amino acid & Annotation & Reference \\
\hline 31 & Val & Shikimate binding & Walker et al. 2013 \\
32 & Pro & Shikimate binding & Walker et al. 2013 \\
298 & Ala & Shikimate binding & Walker et al. 2013 \\
318 & Ile & Shikimate binding & Walker et al. 2013 \\
376 & Phe & Shikimate binding & Walker et al. 2013 \\
414 & Leu & Shikimate binding & Walker et al. 2013, Lallemand et al. 2012 \\
418 & Leu & Shikimate binding & Walker et al. 2013 \\
38 & Ser & carbonyl group of p- coumaroyl moiety & Walker et al. 2013, Eudes et al. 2016 \\
40 & Tyr & carbonyl group of p- coumaroyl moiety & Walker et al. 2013, Lallemand et al. 2012, Eudes et al. 2016 \\
384 & Trp & carbonyl group of p- coumaroyl moiety & Walker et al. 2013 \\
163 & His & carbonyl group of shikimate moiety and & Walker et al. 2013, \\
& & Callemand et al. 2012, Eudes et al. 2016 & Walker et al. 2013 \\
369 & Arg & carbonyl group of shikimate moiety & Walker et al. 2013, Eudes et al. 2016 \\
382 & Thr & carbonyl group of shikimate moiety & Walker et al. 2013 \\
\hline 36 & Thr & Catalysis &
\end{tabular}

2 Note: all positions correspond to SbHCT 
